\title{
BOOLEAN RINGS AND COHOMOLOGY
}

A. D. WALLACE

This note is a comment on a paper of Franklin Haimo [1]. A Stone space is a compact totally disconnected Hausdorff space. Let $B(S)$ be the Boolean ring of the Stone space $S$. The multiplication in $B(S)$ is intersection and the addition is symmetric difference. Thus $B(S)$ is isomorphic with the ring of all maps (=continuous functions) $\phi: S \rightarrow I_{2}, I_{2}$ being the integers mod 2 with the discrete topology. Using the Alexander-Kolmogoroff groups (Spanier [2]) it is readily seen that $\phi \in Z^{0}(S)$, the group of 0 -cocycles of $S$ with $I_{2}$ as coefficient group, if and only if $\phi$ is a map. Using ordinary multiplication of functions in $Z^{0}(S)$ it is at once clear that $B(S) \approx H^{0}(S)$, since $Z^{0}(S)$ $=H^{0}(S)$. If $\left\{S_{\lambda}, \pi_{\lambda_{\mu}}\right\}$ is an inverse system of Stone spaces, then inv $\lim S_{\mathrm{\lambda}}$ is a Stone space. Using Steenrod's continuity theorem (see [2]) we have

$$
{ }^{0}\left(\operatorname{inv} \lim S_{\lambda}\right) \approx \operatorname{dir} \lim H^{0}\left(S_{\lambda}\right)
$$

and hence

$$
B\left(\operatorname{inv} \lim S_{\lambda}\right) \approx \operatorname{dir} \lim B\left(S_{\lambda}\right) .
$$

It might be remarked that, since a Stone space $S$ is compact and totally disconnected, $H^{0}(S)=H(S)$, the cohomology ring of $S$, because $H^{n}(S)=0$ for $n>0$.

\section{BIBLIOGRAPHY}

1. Franklin Haimo, Some limits of Boolean algebras, Proceedings of the American Mathematical Society vol. 2 (1951) pp. 566-576.

2. E. H. Spanier, Cohomology theory for general spaces, Ann. of Math. vol. 49 (1948) pp. 407-427.

The Tulane University of Louisiana

Received by the editors September 29, 1952. 